\title{
Trapping of three-dimensional electrons and transition to two-dimensional transport in the three-dimensional topological insulator $\mathrm{Bi}_{2} \mathrm{Se}_{3}$ under high pressure
}

\author{
A. Segura, $,{ }^{1,2},{ }^{*}$ V. Panchal, ${ }^{1,2}$ J. F. Sánchez-Royo, ${ }^{2}$ V. Marín-Borrás, ${ }^{2}$ V. Muñoz-Sanjosé, ${ }^{2}$ P. Rodríguez-Hernández, ${ }^{3}$ \\ A. Muñoz, ${ }^{3}$ E. Pérez-González, ${ }^{3}$ F. J. Manjón, ${ }^{4}$ and J. González ${ }^{5}$ \\ ${ }^{1}$ Instituto de Ciencia de Materiales de la Universidad de Valencia-MALTA Consolider Team, Universitat de València, \\ 46100 Burjassot, Valencia, Spain \\ ${ }^{2}$ Departamento de Física Aplicada, Universitat de València, 46100 Burjassot, Valencia, Spain \\ ${ }^{3}$ MALTA Consolider Team-Departamento de Física Fundamental II, Universidad de La Laguna, La Laguna, Tenerife, Spain \\ ${ }^{4}$ Instituto de Diseño para la Fabricación y Producción Automatizada, MALTA Consolider Team, \\ Universitat Politècnica de València, 46022 Valencia, Spain \\ ${ }^{5}$ DCITIMAC-MALTA Consolider Team, Universidad de Cantabria, Santander, Spain
}

(Received 28 September 2011; revised manuscript received 13 February 2012; published 24 May 2012)

\begin{abstract}
This paper reports an experimental and theoretical investigation on the electronic structure of bismuth selenide $\left(\mathrm{Bi}_{2} \mathrm{Se}_{3}\right)$ up to $9 \mathrm{GPa}$. The optical gap of $\mathrm{Bi}_{2} \mathrm{Se}_{3}$ increases from $0.17 \mathrm{eV}$ at ambient pressure to $0.45 \mathrm{eV}$ at $8 \mathrm{GPa}$. The quenching of the Burstein-Moss effect in degenerate samples and the shift of the free-carrier plasma frequency to lower energies reveal a quick decrease of the bulk three-dimensional (3D) electron concentration under pressure. On increasing pressure the behavior of Hall electron concentration and mobility depends on the sample thickness, consistently with a gradual transition from mainly 3D transport at ambient pressure to mainly two-dimensional (2D) transport at high pressure. Two-carrier transport equations confirm the trapping of high-mobility 3D electrons, an effect that can be related to a shallow-to-deep transformation of donor levels, associated with a change in the ordering of the conduction band minima. The high apparent areal density and low electron mobility of 2D electrons are not compatible with their expected properties in a Dirac cone. Measured transport parameters at high pressure are most probably affected by the presence of holes, either in an accumulation surface layer or as minority carriers in the bulk.
\end{abstract}

DOI: 10.1103/PhysRevB.85.195139

PACS number(s): 78.20.-e, 71.20.Nr, 72.20.-i

\section{INTRODUCTION}

Bismuth selenide $\left(\mathrm{Bi}_{2} \mathrm{Se}_{3}\right)$ is a low-gap layered chalcogenide that has been extensively investigated for thermoelectric applications. ${ }^{1,2}$ After the prediction of its behavior as a three-dimensional (3D) topological insulator (TI), along with $\mathrm{Bi}_{2} \mathrm{Te}_{3}$ and $\mathrm{Sb}_{2} \mathrm{Te}_{3},{ }^{3}$ research has intensively focused on the characterization of two-dimensional (2D) electrons in the surface Dirac cone. Applications of this topologically protected 2D system to spintronics and quantum computing ${ }^{4,5}$ depend critically on the full understanding of its transport properties. The existence of the Dirac cones with their linear $\mathrm{E}(\mathrm{k})$ dispersion has been conclusively confirmed by photoemission $(\mathrm{PE})^{6-8}$ and scanning tunneling microscopy (STM). ${ }^{9,10}$ Transport parameters of 2D electrons are more difficult to measure because $\mathrm{Bi}_{2} \mathrm{Se}_{3}$ transport properties (as well as free-carrier-related optical properties) are controlled by high-mobility $3 \mathrm{D}$ electrons in the bulk. Some attempts to lower the $3 \mathrm{D}$ electron concentration by playing with the $\mathrm{Bi} / \mathrm{Se}$ ratio in the melt ${ }^{11}$ or by $\mathrm{Ca}$ doping ${ }^{12}$ resulted in high-quality samples with an enhanced 3D electron mobility, masking 2D electrons transport more efficiently. These results led Butch et al. ${ }^{11}$ to propose the existence of a strong surface electron scattering mechanism dramatically reducing the $2 \mathrm{D}$ electron mobility. The most successful strategies to reduce $3 \mathrm{D}$ electron concentration have explored the effect of cation or anion substitution, combined with growth condition optimization. In the related compound $\mathrm{Bi}_{2} \mathrm{Te}_{2} \mathrm{Se}$, Zhi Ren et al. ${ }^{13}$ have managed to grow samples in which the contribution of high-mobility electrons in the Dirac cones is up to $6 \%$ of the sample conductance and can be unambiguously identified as a $2 \mathrm{D}$ contribution to
Shubnikov-de Haas oscillations (SdHOs). Following previous results on the alloy $\mathrm{Bi}_{2-x} \mathrm{Sb}_{x} \mathrm{Se}_{3},{ }^{14}$ Analytis et al. ${ }^{15}$ have grown samples with electron concentrations as low as $\mathrm{n}=$ $2.3 \times 10^{16} \mathrm{~cm}^{-3}$ and, also using SdHOs, have shown that SdHOs at the highest magnetic field are contributed only by 2D electrons in the Dirac cones, because 3D electrons are in the quantum limit, where all of them are collapsed to the lowest Landau level. A third strategy consisted of reducing the $3 \mathrm{D}$ electron contribution to the sample conductance by using very thin samples, down to few quintuple layers (QLs). Bansal et al. ${ }^{16}$ systematically measured the transport properties of samples with thickness from 2 to 2750 QLs, with a bulk 3D electron concentration $\mathrm{n}=1.6 \times 10^{18} \mathrm{~cm}^{-3}$. In this way they identified the TI surface layer contribution to conductance as a thickness-independent sheet electron density of $1.5 \times$ $10^{13} \mathrm{~cm}^{-2}$ and mobility of the order of $1000 \mathrm{~cm}^{2} / \mathrm{Vs}$ for the thicker samples. For a few QLs the TI electron mobility falls to about $300 \mathrm{~cm}^{2} / \mathrm{Vs}$, as the interaction between TI surface layers opens a new scattering channel. PE measurements ${ }^{17,18}$ have also shown that this interaction leads to the opening of a band gap in the 2D system and, consequently, the loss of the linear $\mathrm{E}(\mathrm{k})$ dispersion. Air oxidation of $\mathrm{Bi}_{2} \mathrm{Se}_{3}$ surfaces, as recently reported by Kong et al., ${ }^{19}$ induces $n$-type surface doping and adds a supplementary difficulty to the problem. Even if the TI state remains protected, ${ }^{20,21}$ this surface doping creates surface accumulation subbands that contribute to surface charge transport.

High-pressure techniques are useful tools in the investigation of the electronic structure of semiconductors, based on the ability to produce continuous and finely tuned changes in the 
electronic structure. In this paper we explore the possibility of isolating the $2 \mathrm{D}$ electron contribution to charge transport by applying hydrostatic pressure, based on the observed increase of $\mathrm{Bi}_{2} \mathrm{Se}_{3}$ band gap under pressure, which could result in a reduction of the $3 \mathrm{D}$ electron mobility or in $3 \mathrm{D}$ electrons being trapped by pressure-induced deep levels. During the preparation of the revised version of this paper, the same strategy was used by Hamlin et al., ${ }^{22}$ showing that the resistivity of $\mathrm{Bi}_{2} \mathrm{Se}_{3}$ samples with electron concentrations of the order of $10^{19} \mathrm{~cm}^{-3}$ increases, in fact, under pressure by nearly one order of magnitude up to $8 \mathrm{GPa}$. In this paper we investigate the transport and optical properties of $\mathrm{Bi}_{2} \mathrm{Se}_{3}$ under hydrostatic pressure and correlate them to pressure-induced changes in its electronic structure, as calculated by means of $a b$ initio methods. Sections II and III are devoted to the experimental and calculation methods, respectively. Sec. IV is devoted to the presentation and discussion of results.

\section{EXPERIMENTAL METHODS}

Single crystals of $n$-type $\mathrm{Bi}_{2} \mathrm{Se}_{3}$ were grown using the Bridgman technique. Samples can be easily cleaved from the ingot and cut into slabs for optical or transport measurements. Samples from two ingots grown in different conditions were used. Samples from the A ingot have an electron concentration of $1.5 \times 10^{18} \mathrm{~cm}^{-3}$ and an optical band gap of $170 \mathrm{meV}$. Samples from the B ingot have an electron concentration of $2.5 \times 10^{19} \mathrm{~cm}^{-3}$ and an optical band gap of $380 \mathrm{meV}$. These values that are consistent with previous reports on the BursteinMoss (BM) shift in $\mathrm{Bi}_{2} \mathrm{Se}_{3}{ }^{12,23}$

For Fourier-transform infrared (FTIR) optical measurements under pressure, we used a home-built FTIR setup operating in the mid-IR region $\left(400-4000 \mathrm{~cm}^{-1}\right)^{24}$ and a large aperture membrane diamond anvil cell (MDAC) ${ }^{25}$ with $500-\mu \mathrm{m}$ culet size IIa diamond anvils. A $250-\mu \mathrm{m}$ hole was pierced in a preindented steel gasket. Pressure was measured using the ruby fluorescence scale ${ }^{26}$ and $\mathrm{KBr}$ was used as a pressure-transmitting medium. Hall-effect and resistivity measurements under pressure were made with steel-belted Bridgman tungsten carbide (WC) anvils with a tip of 27 or $15 \mathrm{~mm}$, up to 6 or $12 \mathrm{GPa}$, respectively. Force on the opposite anvils is applied by a 150-ton oil press. A copper coil around the press piston is used to generate the magnetic field for Hall-effect measurements. Two annealed pyrophyllite gaskets are used to contain the sample in hexagonal $\mathrm{BN}$ pressure-transmitting medium, and silver leads between the gaskets direct the current and voltage signals. The setup and its calibration have been described elsewhere. ${ }^{27,28}$

\section{AB INITIO CALCULATIONS}

$A b$ initio calculations have been performed within the density functional theory (DFT) ${ }^{29}$ using the plane-wave method and the pseudopotential theory with the VASP package ${ }^{30}$ with the projector-augmented wave scheme $(\mathrm{PAW})^{31}$ and spin-orbit (SO) coupling. A basis set, including plane waves up to an energy cutoff of $320 \mathrm{eV}$, was used in order to achieve highly converged results. We have used the generalized-gradient approximation (GGA) for the exchange-correlation energy with the PBEsol ${ }^{32}$ prescription. At each selected volume, the structures were fully relaxed to their equilibrium configuration through the calculation of the forces on atoms and the stress tensor with a dense special $k$-point sampling. The application of DFT-based calculations to the study of semiconductor properties under high pressure has been reviewed in Ref. 33.

\section{RESULTS AND DISCUSSION}

\section{A. FTIR absorbtion and reflection results}

We will first present and discuss the optical results, because they can be more directly related to the semiconductor band structure.

Figure 1 shows the optical transmittance and reflectance spectra at ambient pressure for the two $\mathrm{Bi}_{2} \mathrm{Se}_{3}$ samples that have been used in this work. Electron concentrations are $1.5 \times 10^{18}$ and $2.5 \times 10^{19} \mathrm{~cm}^{-3}$ for samples $\mathrm{A}$ and $\mathrm{B}$, respectively. The BM shift is clearly seen as well as the plasma reflection structure for sample B. A Drude model fit is also shown [see Eq. (1)], corresponding to a free-carrier plasma frequency of $730 \mathrm{~cm}^{-1}$ and a damping parameter of $120 \mathrm{~cm}^{-1}$. Spectra near the fundamental absorption edge are dominated by large interferences, as a result of the high value of the refractive index (about 5.5). ${ }^{34}$ The interference fringe amplitude decreases at low photon energy due to the onset of free-carrier absorption and at high photon energies due to the fundamental absorption edge (band-to-band absorption).

Figures 2(a) and 2(b) show the optical transmittance inside the DAC for samples A [Fig. 2(a)] and B [Fig. 2(b)] at two

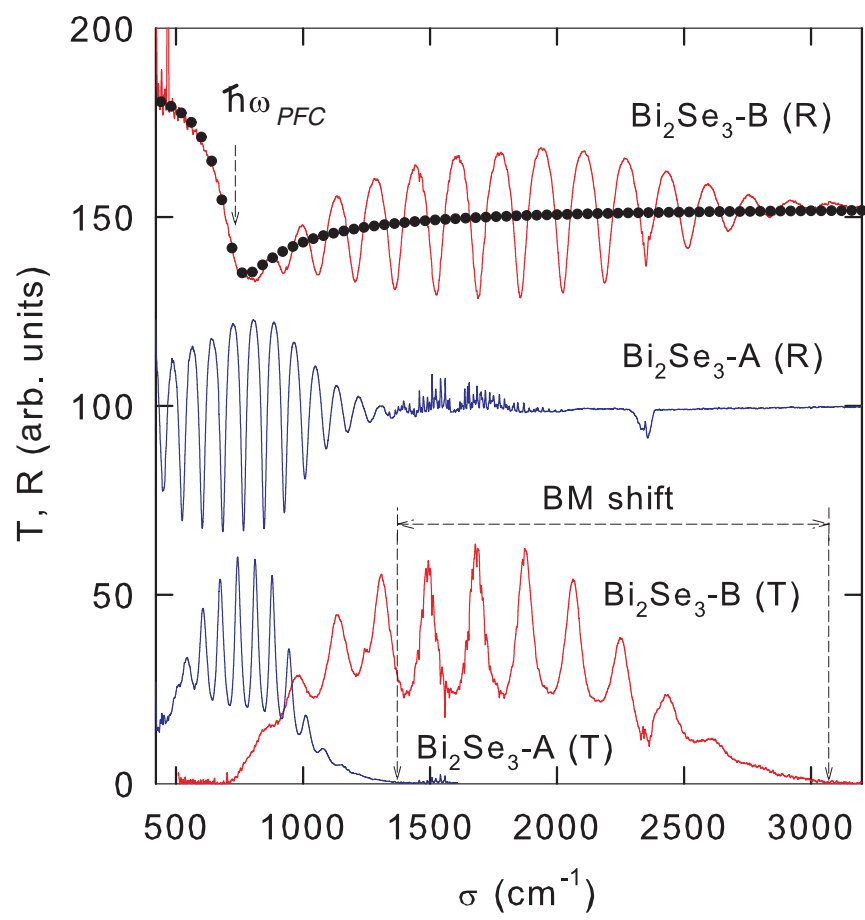

FIG. 1. (Color online) Experimental reflectance (R) and transmittance (T) outside the DAC two $\mathrm{Bi}_{2} \mathrm{Se}_{3}$ samples from ingots $\mathrm{A}$ [blue (medium gray) lines] and B [red (dark gray) lines]. The BM shift is indicated by the interval between the vertical dashed lines marking the optical gaps for samples A and B. Reflectance spectra have been vertically shifted for clarity. Black line and points: Drude model plasma reflection fit to reflectance of sample B. 

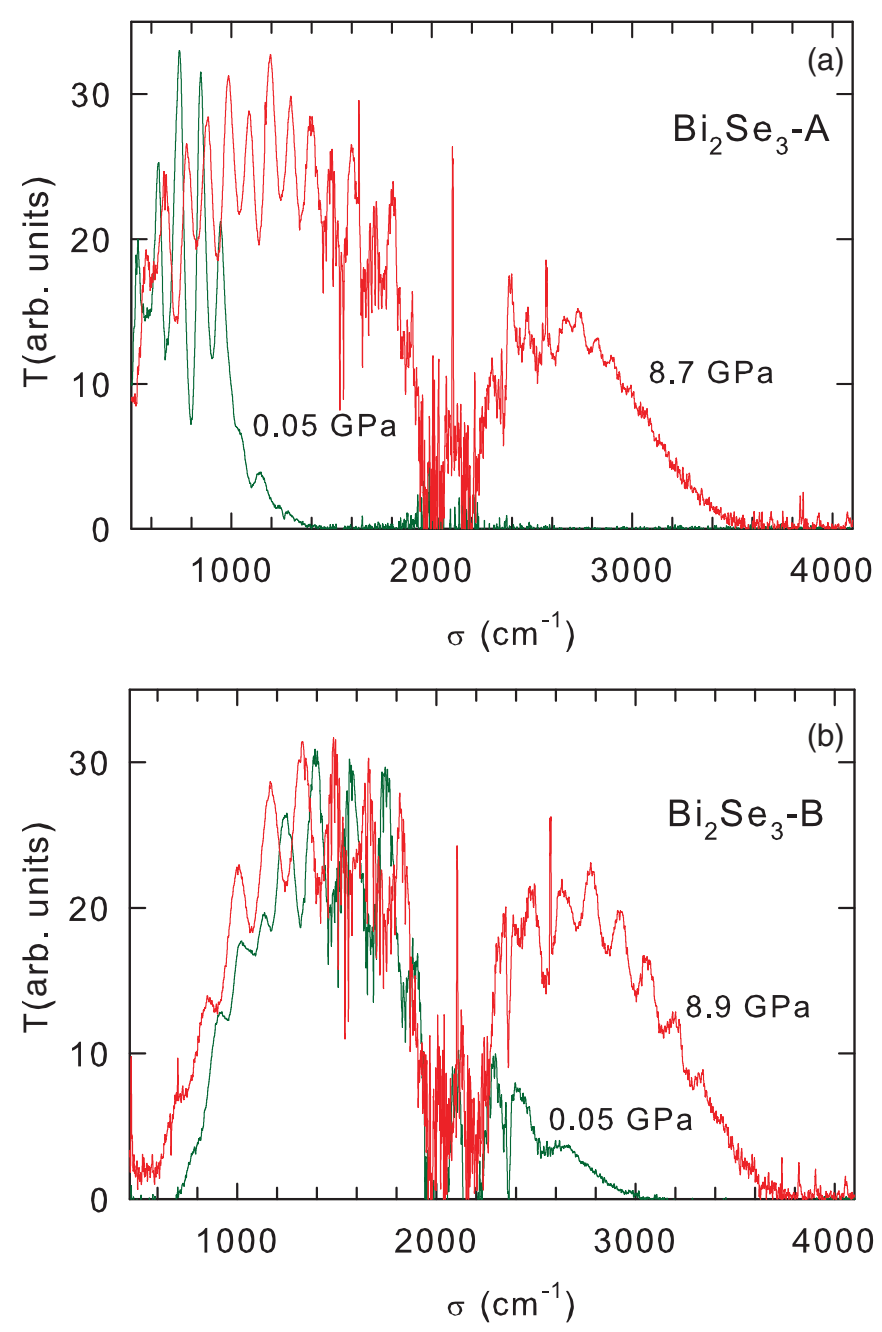

FIG. 2. (Color online) (a) Optical transmittance inside the DAC for a sample from the A ingot at two pressures. (b) The same for a sample from ingot B.

different pressures. Owing to the large interference pattern, the absorption coefficient can be accurately determined from transmittance spectra only in a small photon energy range between the photon energy at which the interference pattern vanishes and the photon energy at which the transmitted intensity merges into noise. This limitation prevents a detailed analysis of the absorption edge shape. As we are interested only in the pressure dependence of the band gap, we define an optical gap as the photon energy at which transmittance is $1 \%$ with respect to the transmittance at the interference maxima in the transparency interval. This value of the transmittance is arbitrary. It was chosen because it corresponds to the highest absorption coefficient that can be accurately determined from transmitted energy levels larger than the spectrometer noise level. Given a sample thickness about $5 \mu \mathrm{m}$, the optical gap so defined corresponds to the photon energy at which the absorption coefficient reaches a value of $10^{4} \mathrm{~cm}^{-1}$. The optical gap so defined for sample A, $170 \pm 5 \mathrm{meV}$, turns out to be very close the reported intrinsic value of the $\mathrm{Bi}_{2} \mathrm{Se}_{3}$ direct gap, $160 \pm 10 \mathrm{meV},{ }^{23}$ but slightly higher as expected owing to a small BM shift. ${ }^{12,23}$ The pressure dependence so determined corresponds to the pressure shift of the absorption

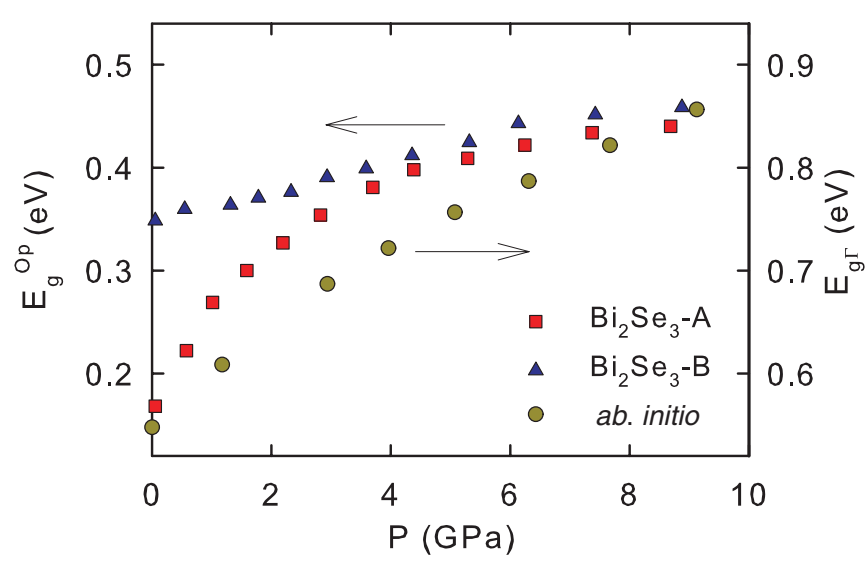

FIG. 3. (Color online) Pressure dependence of the optical gap for sample A (squares), B (triangles; left-axis), and ab initio calculated band gap at the $\Gamma$ point (circles; right axis).

edge at the constant absorption coefficient. Figure 3 shows the pressure dependence of the optical gap for both samples. The optical gap of sample A quickly increases under pressure, with a pressure coefficient of about $100 \mathrm{meV} / \mathrm{GPa}$ up to $1 \mathrm{GP}$, and then nonlinearly tends to $450 \mathrm{meV}$ at $8 \mathrm{GPa}$. The optical gap of sample B exhibits a more linear pressure dependence and a much lower pressure coefficient (16 meV/GPa). Above $4 \mathrm{GPa}$, the optical gap and its pressure dependence become virtually identical for both samples, strongly suggesting that the BM shift and, consequently, the free-electron concentration decrease under pressure.

Figure 4 shows the reflectance spectrum of a thin slab from the B sample at different pressures. Besides the alreadymentioned increase of the optical gap, a shift of the free-carrier plasma reflection structure to lower energies is clearly visible, as shown by the Drude model fits to three experimental spectra. At pressures above $4 \mathrm{GPa}$, the plasma reflection structure is below the lower limit of our spectrometer range. Nevertheless, in such a thin sample $(2.3 \mu \mathrm{m})$, the orders of the reflection minima can be unambiguously assigned. Using the refractive index dispersion at ambient pressure, ${ }^{34}$ the sample thickness can be also determined. $\mathrm{Bi}_{2} \mathrm{Se}_{3}$ cell parameters under pressure have been recently measured. ${ }^{35}$ We can obtain the sample thickess at each pressure from the pressure dependence of the $c$ parameter and then determine the refractive index spectrum as a function of pressure, as shown in Fig. 5. The free-carrierrelated structure is the refractive index drop observed at low frequency. This feature clearly shifts to lower frequencies as pressure increases, consistent with the observed decrease of the plasma frequency.

Results shown in Fig. 5 exhibit another important feature that seems relevant to notice and discuss, namely, the large overall increase of the refractive index under pressure. In the region between 2000 and $3000 \mathrm{~cm}^{-1}$, the refractive index increases by more than $22 \%$ between ambient pressure and $9 \mathrm{GPa}$, which corresponds to an increase of the electronic dielectric constant by more than $44 \%$. About $15 \%$ of this increase is simply related to the volume decrease ${ }^{35}$ under pressure. The remaining increase (about 30\%) should come from the increase of the electronic polarizability. The $\mathrm{Bi}_{2} \mathrm{Se}_{3}$ electronic dielectric function at ambient pressure has been 


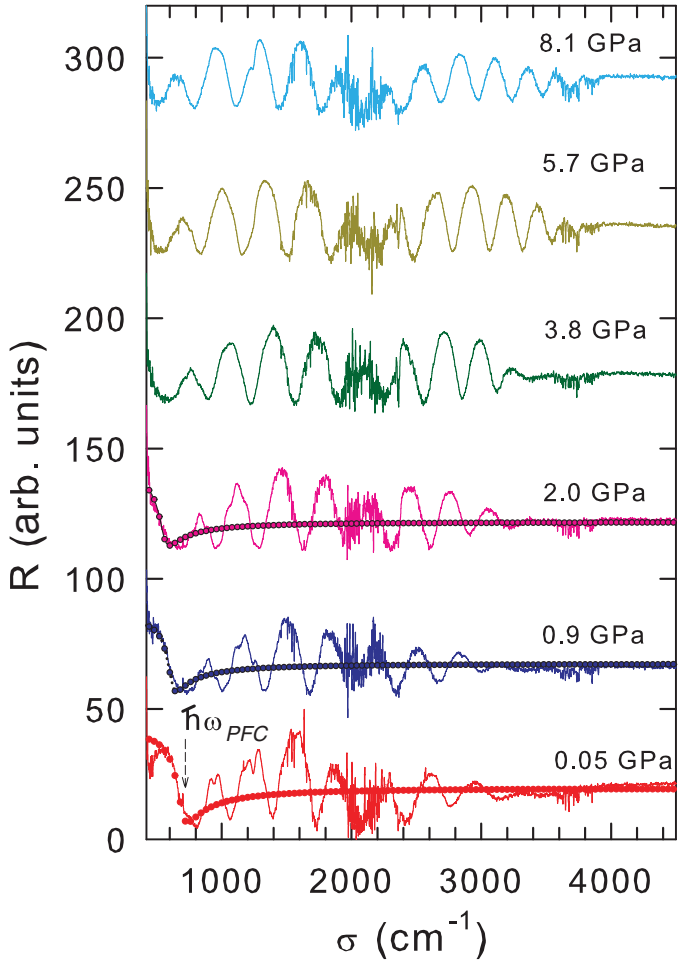

FIG. 4. (Color online) Reflectance spectra of a $2.3-\mu \mathrm{m}$-thick sample from the B ingot at the indicated pressures. Spectra have been vertically shifted for clarity. Dotted lines: Drude plasma reflection fits to experimental spectra.

shown to be mainly determined ${ }^{36,37}$ by a very intense optical transition at $2 \mathrm{eV}$ that would correspond to its Penn gap, ${ }^{38}$ according to the Phillips-Van Vechten mode ${ }^{39-41}$ for the electronic polarizabiliy of semiconductors. Continuous lines in Fig. 5 have, then, been calculated with a Phillips-Van Vechten term for the valence electron contribution and a Drude model

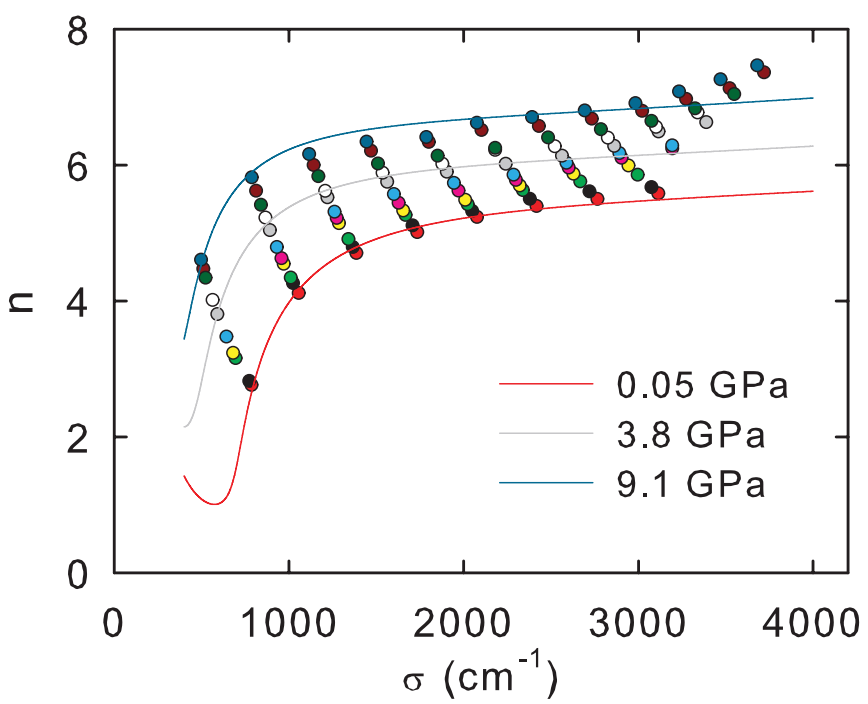

FIG. 5. (Color online) Symbols: experimental refractive index spectrum at different pressures as calculated from interference minima. Dotted lines: Drude model refractive index fits. for the free-carrier contribution

$$
\begin{aligned}
\varepsilon(\omega) & =[n(\omega)]^{2}=1+\frac{\omega_{P V}^{2}}{\omega_{0}^{2}-\omega^{2}}-\frac{\varepsilon_{e} \omega_{P F C}^{2}}{\omega(\omega+i \gamma)}, \\
\varepsilon_{e} & =1+\frac{\omega_{P V}^{2}}{\omega_{0}^{2}},
\end{aligned}
$$

where the Phillips-Van Vechten term includes the plasma frequency of valence electrons $\left(\omega_{P V}\right)$ and the Penn gap $\left(\hbar \omega_{0}\right)$, and the Drude term includes the electronic dielectric constant $\left(\varepsilon_{e}\right)$, the free-carrier plasma frequency $\left(\omega_{P F C}\right)$, and the free-carrier damping frequency $(\gamma$; inverse of the relaxation time). At ambient pressure the Penn gap has been taken to be equal to $2 \mathrm{eV},{ }^{36,37}$ and the valence electron plasma frequency has been chosen to give the right value of the electronic dielectric constant $\left(\varepsilon_{e}=29.5\right) .{ }^{34}$ The pressure dependence of $\omega_{P V}$ is taken into account through the measured $\mathrm{Bi}_{2} \mathrm{Se}_{3}$ compressibility. ${ }^{35}$ Then, the only fitting parameters are $\omega_{0}$, $\omega_{P F C}$, and $\gamma$. They can be determined independently with good accuracy as they affect different features of the spectrum. The refractive index experimental values are larger than the calculated ones near the optical gap due to the contribution of the direct fundamental transition, not taken into account in Eq. (1). The Penn gap decreases nonlinearly from $2 \mathrm{eV}$ at ambient pressure to $1.86 \mathrm{eV}$ at $3.8 \mathrm{GPa}$ and $1.74 \mathrm{eV}$ at $9.1 \mathrm{GPa}$. This decrease of the Penn gap is at the origin of the large increase of $\mathrm{Bi}_{2} \mathrm{Se}_{3}$ electronic polarizability under high pressure.

Figure 6(a) shows the pressure dependence of the freecarrier plasma frequency as obtained from the reflectivity and refractive index Drude fits. The decrease of the plasma frequency is consistent with the previously mentioned quenching of the BM shift. This effect is also illustrated in Fig. 6(a) through the decrease of the difference between the optical gaps of samples $\mathrm{B}$ and $\mathrm{A}$ as pressure increases. In the degenerate regime the electron concentration is proportional to $\left(\mathrm{E}_{\mathrm{F}}-\mathrm{E}_{\mathrm{C}}\right)^{3 / 2}$ [Fermi level measured from the conduction-band minimum (CBM)]. The Fermi-level shift can be reasonably assimilated to the BM shift, as obtained from the optical gap difference between samples B and A. On the other side, the electron concentration is proportional to the square of the plasma frequency. In Fig. 6(b) we plot $\left(\Delta \mathrm{E}_{\mathrm{g}}\right)^{3 / 2}$ versus $\left(\hbar \omega_{\mathrm{PFC}}\right)^{2}$. As both quantities are proportional to the electron concentration, the linear relationship between them consistently confirms the decrease of the electron concentration under pressure.

\section{B. Electronic structure under high pressure}

Let us discuss the above-mentioned results in light of ab initio calculations. Figure 7 shows the calculated band structure of $\mathrm{Bi}_{2} \mathrm{Se}_{3}$, including spin-orbit effects. At ambient pressure, our results are virtually identical to those reported by Larson $e$ t al ${ }^{42}$ and Zhang $e t$ al.,${ }^{3}$ except that the latter authors do not show the band dispersion in the $\Gamma$-U direction. $\mathrm{Bi}_{2} \mathrm{Se}_{3}$ is an indirect semiconductor, with the valence-band maximum (VBM) located halfway in the $\Gamma-\mathrm{U}$ direction and the CBM at the $\Gamma$ point. Orbital composition at ambient pressure reveals the band inversion at the $\Gamma$ point that is at the origin of the TI 2D system at the surface, as discussed in depth by Zhang et al. ${ }^{3}$ At the $\Gamma$ point, the CBM wave function has mainly the Se $p$ character, while the VBM wave function has mainly the Bi $p$ 

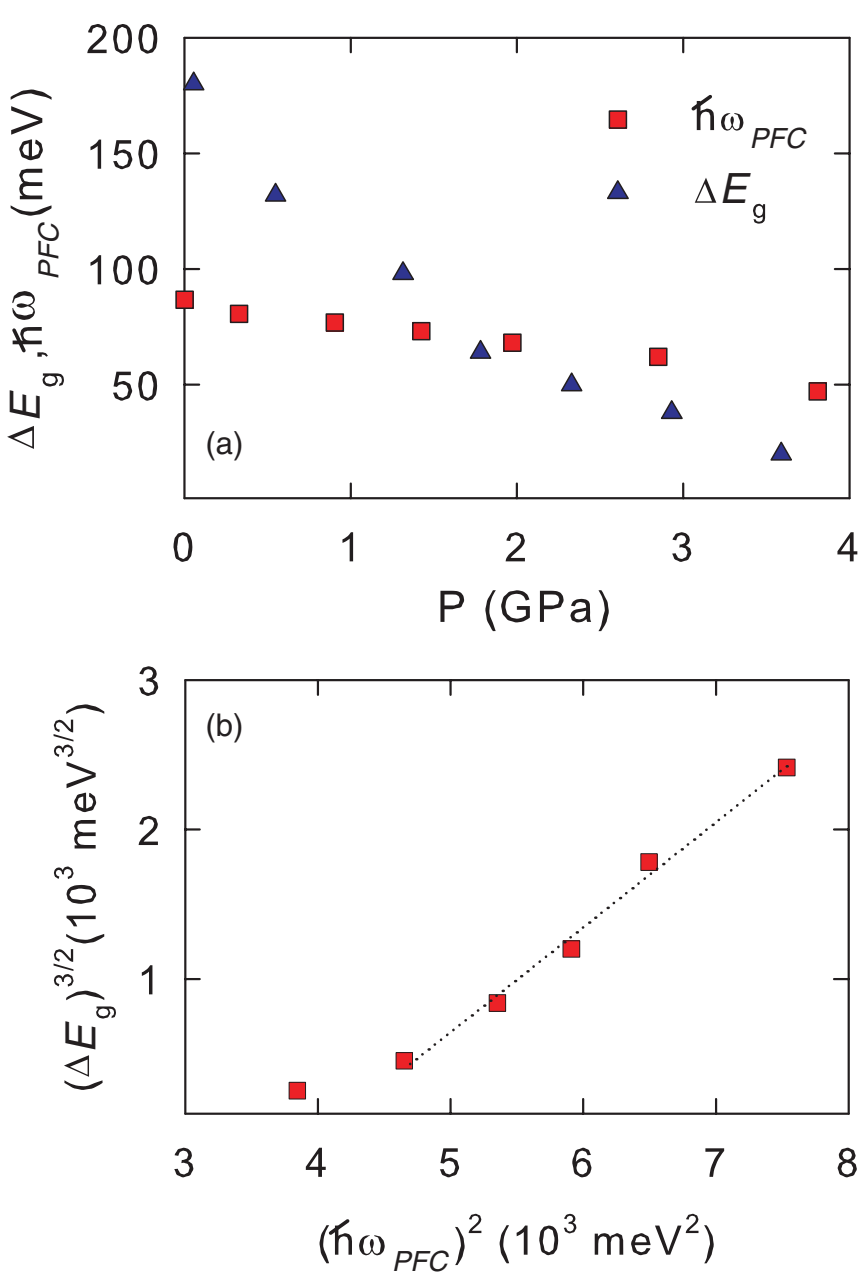

FIG. 6. (Color online) (a) Filled triangles: pressure dependence of the free-carrier plasma frequency in a sample from the B ingot, as obtained from the reflectivity and refractive index fits. Filled squares: pressure dependence of the optical gap difference between samples from ingots B and A. (b) Linearity test of the free-electron concentration as estimated from the free-carrier plasma frequency (horizontal axis) and BM shift (vertical axis).

and Se $s$ characters. The orbital composition does not basically change under pressure. This is a relevant result indicating that the TI character should be stable under pressure. The sample environment in our high-pressure experiments involves interfaces between the material and the pressure-transmitting medium ( $\mathrm{KBr}$ for optical and $\mathrm{BN}$ for transport experiments). These interfaces are not basically different from those between the material and vacuum, as shown by Chang et al., ${ }^{43}$ and then preserve the surface TI states.

The observed increase of the optical gap is consistent with the increase of the direct gap at the $\Gamma$ point, as it corresponds to the only electric-dipole-allowed transition for photon energies around the forbidden band. Calculated values are larger than experimental ones, as shown in Fig. 3. This overestimation is a result of using the GGA approximation. The calculated linear coefficient of the $\Gamma$ direct transition at low pressure, about $60 \mathrm{meV} / \mathrm{GPa}$, is lower than the measured one, but the overall increase of the $\Gamma$ band gap in the explored pressure range $(300 \mathrm{meV})$ is very close to the experimental value

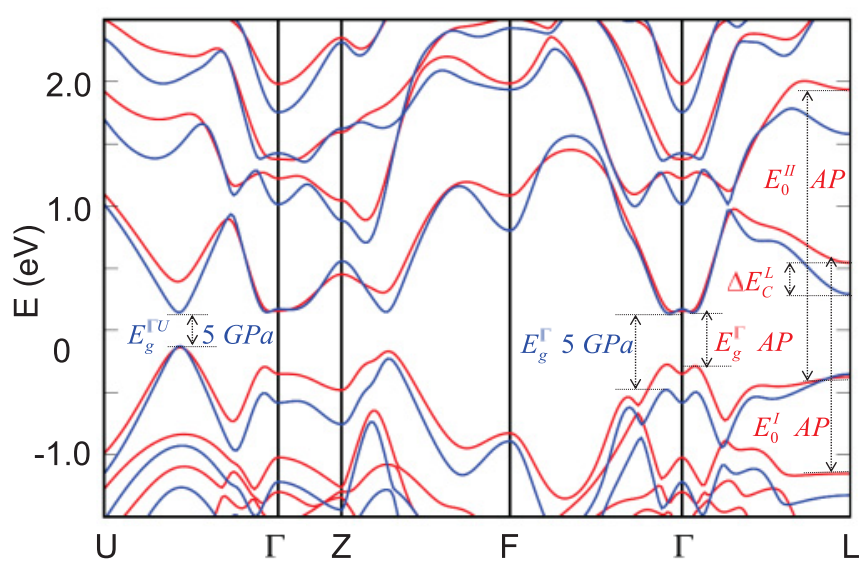

FIG. 7. (Color online) Calculated band structure of $\mathrm{Bi}_{2} \mathrm{Se}_{3}$ in the gap region, including spin-orbit interaction, at two pressures. Notation for the Brillouin zone points is the same as in Refs. 3 and 42.

(270 meV). Let us notice that theoretical points in Fig. 3 correspond to the gap at the $\Gamma$ point. The spin-orbit interaction creates the typical "camel's back" structure shifting band absolute extrema slightly away from the $\Gamma$ point. As a consequence, the actual calculated band gap would be about $10 \%$ smaller.

It is also relevant to stress that several minima (maxima) of the conduction (valence) band shift to lower (higher) energies under pressure and, in particular, the ones in the $\Gamma-\mathrm{U}$ direction become the absolute minimum (maximum) above $5 \mathrm{GPa}$.

Free-carrier freeze-out under pressure, as observed in III-V ${ }^{44}$ or III-VI ${ }^{45}$ semiconductors, has been related to a shallow-to-deep transformation of donor levels. More complex situations can arise in the case of metastable states with local bond distortions, but, in a simplified approach, we can consider that donor levels associated with a low effective mass CBM, like the $\Gamma$ minimum in III-V semiconductors, are shallow, while donor levels associated with a subsidiary minimum with a higher effective mass are, in general, deep. If one of those minima shifts down in energy, its related deep donor becomes deeper than the shallow level and traps free electrons as it enters the forbidden band (even if the related minimum does not become the absolute CBM). In the case of $\mathrm{Bi}_{2} \mathrm{Se}_{3}$, the most likely candidate to have an associated deep level seems to be the minimum at the $\mathrm{L}$ point, as it has the lowest curvature (and then the highest effective mass) and shifts to lower energies at a rate of some $-52 \mathrm{meV} / \mathrm{GPa}$.

Concerning the increase of the electronic dielectric constant, let's first try to identify the electronic transitions corresponding to the Penn gap (dielectric function peak at $2 \mathrm{eV}){ }^{36,37}$ For optical transitions between the valence and conduction band, the only area of the band structure that could give rise to a high joint density of states at $2 \mathrm{eV}$ seems to be around the $\Gamma$-L direction. In this region two transitions could contribute to the Penn gap, (i) the transition between the second valence band and the first conduction band along most of the $\Gamma$-L direction, excluding an interval close to the $\Gamma$ point $\left(\mathrm{E}_{0}^{\mathrm{I}}\right.$ in Fig. 7) and (ii) the transition between the valence band and the second conduction band near the $\mathrm{L}$ point ( $\mathrm{E}_{0}^{\mathrm{II}}$ in Fig. 7). Then, the Penn gap of $\mathrm{Bi}_{2} \mathrm{Se}_{3}$ could be reasonably related to these transitions as, apart from being in the right energy range, band structure calculations predict for both of them a decrease 

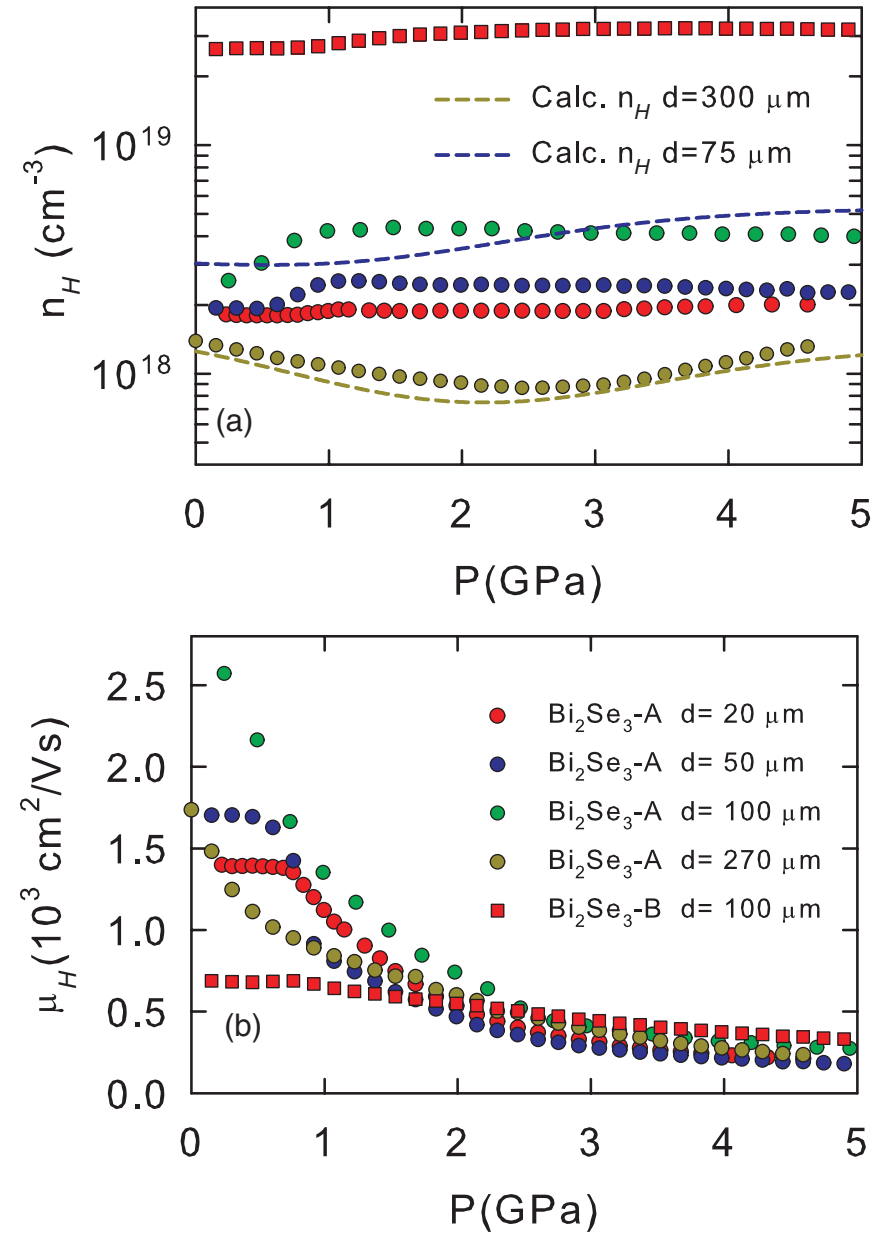

FIG. 8. (Color online) (a) Symbols: pressure dependence of the Hall electron concentration for several $\mathrm{Bi}_{2} \mathrm{Se}_{3}$ samples with different thicknesses. Broken lines: calculated Hall electron concentration using Eq. (2) with two different thickness value. (b) Pressure dependence of the Hall electron mobility for several $\mathrm{Bi}_{2} \mathrm{Se}_{3}$ samples.

of the transition energy under pressure (more the case for for $\mathrm{E}_{0}^{\mathrm{II}}$ ) with a pressure coefficient close to the one experimentally determined from the refractive index pressure behavior.

\section{Transport properties under pressure}

We will now present and discuss the results of transport experiments under pressure. Figure 8 shows the pressure dependence of Hall electron concentration (a) and mobility (b) for several $\mathrm{Bi}_{2} \mathrm{Se}_{3}$ samples. In contrast to the free-carrier freeze-out detected through FTIR spectroscopy, transport experiments indicate that the pressure behavior of the Hall electron concentration $\left(n_{H}\right)$ depends on the sample thickness. For thin samples $(\mathrm{d}<150 \mu \mathrm{m})$ from the A ingot, $n_{H}$ slightly increases up to around $1 \mathrm{GPa}$ and then remains virtually constant up to $5 \mathrm{GPa}$. For thick samples $(\mathrm{d}>200 \mu \mathrm{m})$, the electron concentration decreases under pressure up to 3 $\mathrm{GPa}$ and then slightly increases. More dramatic (and also thickness-dependent) changes are observed in the electron Hall mobility. For thin samples the Hall mobility remains nearly constant up to $1 \mathrm{GPa}$ and then gradually decreases from its ambient pressure value $\left(1700 \mathrm{~cm}^{2} / \mathrm{Vs}\right)$ to about $200 \mathrm{~cm}^{2} / \mathrm{Vs}$ at $5 \mathrm{GPa}$. For thick samples, the Hall mobility decreases monotonously from very low pressure. For samples from the $\mathrm{B}$ ingot, the behavior of transport parameters is similar, but Hall electron concentrations are larger, and Hall mobilities are smaller than the ones measured in the samples from ingot A. Hall mobility converges to similar values, for all samples, at about $5 \mathrm{GPa}$. Results for samples from the B ingot are close to those obtained by Hamlin et al., ${ }^{22}$ using samples with very close electron concentrations.

No bulk mechanism (simple trapping of 3D electrons or electron transfer to a low-mobility CBM) can give account of the observed thickness sensitivity of the pressure behavior of $\mathrm{Bi}_{2} \mathrm{Se}_{3}$ transport parameters. This behavior is typical of the coexistence of 3D electron transport in the bulk and 2D electron transport in the surface of a sample. A homogeneous distribution of 2D electrons bound to planar interlayer impurity distributions, as observed in layered InSe, ${ }^{45}$ would neither explain the present results. In InSe, planar defects are homogeneously distributed across the sample and do not produce macroscopic thickness sensitivity. Let us use a two-carrier model, in which the bulk electron concentration (mobility) is $n_{3 D}\left(\mu_{3 D}\right)$, and the areal density (mobility) of $2 \mathrm{D}$ carriers at the sample surface is $\mathrm{n}_{\mathrm{S}}\left(\mu_{2 D}\right)$. The effective $3 \mathrm{D}$ transport parameters for a sample with thickness $d$, as they would be measured from resistivity and Hall-effect experiments, would be given by

$$
\begin{aligned}
\sigma & =e\left(n_{3 D} \mu_{3 D}+\frac{n_{S}}{d} \mu_{2 D}\right), \quad \mu_{H}=\frac{n_{3 D} \mu_{3 D}^{2}+\frac{n_{S}}{d} \mu_{2 D}^{2}}{n_{3 D} \mu_{3 D}+\frac{n_{S}}{d} \mu_{2 D}}, \\
n_{H} & =\frac{1}{e R_{H}}=\frac{\left(n_{3 D} \mu_{3 D}+\frac{n_{S}}{d} \mu_{2 D}\right)^{2}}{n_{3 D} \mu_{3 D}^{2}+\frac{n_{S}}{d} \mu_{2 D}^{2}} .
\end{aligned}
$$

From these equations an effective carrier concentration $n^{*}$ can be defined as

$$
\begin{gathered}
n^{*}=\frac{\sigma}{e \mu_{3 D}^{2}} \mu_{H}=n_{3 D}+\frac{n_{S}}{d} \frac{\mu_{2 D}^{2}}{\mu_{3 D}^{2}}=n_{3 D}+n_{2 D}^{*}, \\
n_{3 D}=n^{*}-n_{2 D}^{*} .
\end{gathered}
$$

If we assume that the 3D electron mobility is weakly dependent on pressure, $n^{*}$ can be determined from experimental results by setting $\mu_{3 D}$ as the Hall mobility at ambient pressure. Figure 9(a) shows $n^{*}$ as a function of pressure for several samples. It exponentially decreases under pressure and tends to a value inversely correlated to the sample thickness, which is consistent with Eq. (2). We can estimate $n_{2 D}^{*}$ from the limit value of $n^{*}$ at high pressure and then plot $n_{3 D}=n^{*}-n_{2 D}^{*}$ as a function of pressure [Figure 9(b)]. In this way the exponential decrease of $n_{3 D}$ is more clearly put in evidence. This exponential decrease is fully consistent with the trapping of 3D electrons by a deep level shifting linearly to lower energies. With a single donor model and no acceptor compensation, the deep trap pressure coefficient would be $-65(5)$ and $-30(3) \mathrm{meV} / \mathrm{GPa}$ for samples from ingots $\mathrm{A}$ and $\mathrm{B}$, respectively. This result is consistent with the pressure decrease of the plasma frequency shown in Fig. 6(a). The decrease of $n_{3 D}$ in sample $\mathrm{B}$ by a factor 4 between ambient pressure and $4 \mathrm{GPa}$ corresponds to the decrease of the plasma frequency by a factor 2 through the same pressure range. 

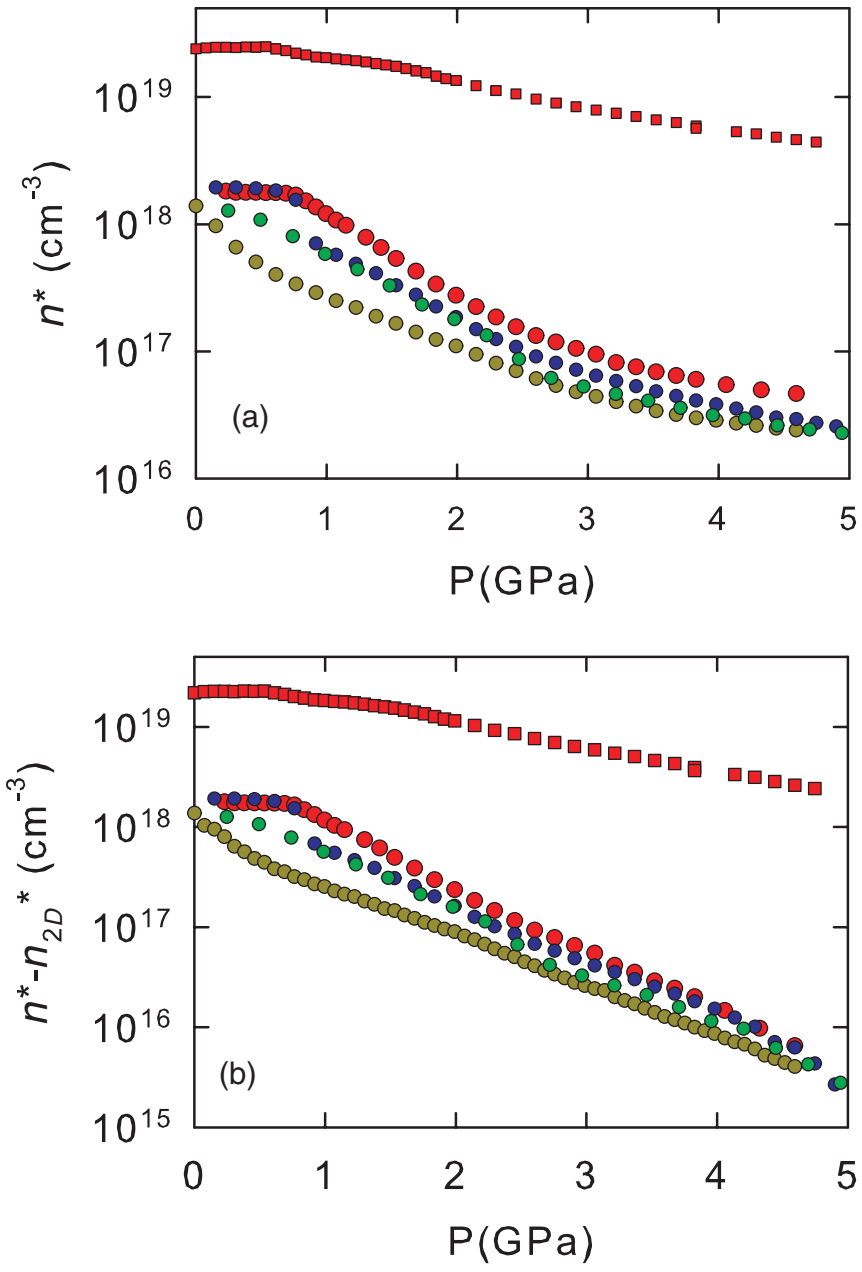

FIG. 9. (Color online) (a) Pressure dependence of the effective electron concentration $n^{*}$ for several samples as calculated using Eq. (2). (b) Pressure dependence of the effective $n_{3 D}$ concentration as calculated using Eq. (3).

The thickness sensitivity of the transport parameters pressure behavior can be understood as a result of the relative weight of 3D and 2D transport parameters and their change under pressure. For very thick samples at ambient pressure, $n_{3 D}$ is larger than $\mathrm{n}_{\mathrm{s}} / \mathrm{d}$, and transport parameters at ambient pressure are dominated by $3 \mathrm{D}$ electrons. As pressure increases, 3D electrons are trapped, and charge transport will become gradually dominated by $2 \mathrm{D}$ electrons. For thin samples the situation is such that the effective $2 \mathrm{D}$ electron concentration is actually larger than the $3 \mathrm{D}$ one, but $3 \mathrm{D}$ electrons determine the Hall voltage owing to its larger mobility. Dotted lines in Fig. $8(\mathrm{a})$ are calculated with the same parameters $\left(\mu_{3 D}=\right.$ $1600 \mathrm{~cm}^{2} / \mathrm{Vs}, \mu_{2 D}=270 \mathrm{~cm}^{2} / \mathrm{Vs}, n_{3 D}=1.8 \times 10^{18} \mathrm{~cm}^{-3}$, and $n_{S}=4.0 \times 10^{16} \mathrm{~cm}^{-2}$ ), assuming that 3D electrons are trapped by a deep level shifting down in energy at $65 \mathrm{meV} / \mathrm{GPa}$, and simply changing the sample thickness from 300 (lower line) to $75 \mu \mathrm{m}$ (upper line). This calculation is not intended as an exact fit to the experimental results. It is just an illustration, with a very simple model, of the interplay between both types of transport and the way it changes under high pressure, depending on the sample thickness.
A surprising result of this analysis is the high Hall areal concentration of 2D electrons, $4 \times 10^{16}$ and $4 \times 10^{17} \mathrm{~cm}^{-2}$ for samples from the A and B ingots, respectively. These areal concentrations are about two to three orders of magnitude larger than the ones expected in a Dirac cone. From the linear dispersion curves obtained by angle-resolved $\mathrm{PE},{ }^{6-8}$ an elementary calculation leads to a $2 \mathrm{D}$ electron areal density of the order of $\mathrm{k}_{\mathrm{F}}^{2}$. For degenerate samples, like those from the $\mathrm{B}$ ingot used here, $\mathrm{k}_{\mathrm{F}} \sim 0.1 \AA^{-1}$ and the areal electron concentration at the surface would be about $10^{14} \mathrm{~cm}^{-2}$. For samples from the $\mathrm{A}$ ingot, if we estimate $\mathrm{k}_{\mathrm{F}}$ from the Fermi-level shift between the A and B samples, its value would be $0.05 \AA^{-1}$, corresponding to an areal density of $2.5 \times$ $10^{13} \mathrm{~cm}^{-2}$.

Anomalously high Hall electron concentrations in low gap semiconductors are a result of the presence of free holes that partially compensate the electron Hall-effect voltage. The existence of a 2D electron system at the surface of an extrinsic $n$-type semiconductor originates band bending and a depletion zone close to the surface, as $2 \mathrm{D}$ electrons are compensated by ionized donors. From Shubnikov-de Haas and PE experiments, Analytis et al. ${ }^{46}$ have measured a surface barrier of $75 \mathrm{meV}$ between the bulk and the surface in $\mathrm{Bi}_{2} \mathrm{Se}_{3}$ samples with electron concentrations closer to those from the $\mathrm{B}$ ingot here studied. In such a low gap semiconductor, this barrier is about half of the band gap. Shifting the valence band at the surface toward the Fermi level by $75 \mathrm{meV}$ originates a hole accumulation layer.

Let us first discuss if such a hole accumulation layer can explain the observed behavior. If the only contributions to surface conductance $\left(\Sigma_{S}\right)$ are 2D electrons in the Dirac cone and holes in the accumulation layer, then $\Sigma_{S}=e\left(n_{S} \mu_{2 D}+p_{S} \mu_{h}\right)$, where $\mathrm{n}_{\mathrm{S}}$ and $\mu_{2 \mathrm{D}}$ are the areal concentration and mobility of $2 \mathrm{D}$ electrons [as in Eq. (2)] and $\mathrm{p}_{\mathrm{S}}$ and $\mu_{\mathrm{h}}$ are the areal concentration and mobility of holes in the accumulation layer. Typical values of conductance at high pressure are about 2 to 3 and 20-30 $\Omega^{-1}$ for samples from the $\mathrm{A}$ and $\mathrm{B}$ ingots, respectively. If we assume the above-mentioned areal densities for Dirac cone electrons, their contribution to the conductance, even with high electron mobilities, would not be larger than $10^{-2} \Omega^{-1}$. This means that the main contribution to conductance should come from holes. A hole mobility of some $600 \mathrm{~cm}^{2} / \mathrm{Vs}^{47}$ would lead to an unrealistically high hole areal density of about $2 \times 10^{16} \mathrm{~cm}^{-2}$, far too large if one considers that the Debye length in $\mathrm{Bi}_{2} \mathrm{Se}_{3}$ would be about $100 \mathrm{~nm}$ for the estimated 3D electron concentration at $5 \mathrm{GPa}$.

The change of the band structure under pressure (Fig. 7) provides further hints. On the one side, the absolute VBM and $\mathrm{CBM}$ at $5 \mathrm{GPa}$ are midway in the $\Gamma-\mathrm{U}$ direction, and the band gap is smaller than the $\Gamma$-band gap at ambient pressure. Then, a much larger minority hole concentration is expected. Their mobility can also be larger than it is at ambient pressure, as VBM at the $\Gamma-\mathrm{U}$ direction exhibits higher curvature than the VBM at the $\Gamma$ point at ambient pressure, and a lower effective mass is expected. Trapping of $3 \mathrm{D}$ electrons also shifts the Fermi level toward the center of the forbidden band and increases the minority hole concentration. At high pressure, when most 3D electrons are trapped and minority holes (with electron concentration $p$ and mobility $\mu_{\mathrm{h}}$ ) are excited, the Hall 
mobility and Hall carrier concentration would be given by

$$
\mu_{H}=\frac{\frac{n_{S}}{d} \mu_{2 D}^{2}-p \mu_{h}^{2}}{\frac{n_{S}}{d} \mu_{2 D}+p \mu_{h}}, \quad n_{H}=\frac{\left(\frac{n_{S}}{d} \mu_{2 D}+p \mu_{h}\right)^{2}}{\frac{n_{S}}{d} \mu_{2 D}^{2}-p \mu_{h}^{2}} .
$$

This equation qualitatively explain why the highest Hall electron concentrations [Fig. 8(a)] are measured in thicker samples (except for the thickest one in which the 2D regime is clearly not attained at $5 \mathrm{GPa}$ ). For thick samples the first term in the denominator is smaller than in thin samples, and compensation by holes is more effective. Minority hole concentrations larger than $10^{16} \mathrm{~cm}^{-3}$ are possible for a band gap of some $150 \mathrm{meV} .^{47}$ The hole areal concentration for a $100-\mu$ m-thick sample would then be of $10^{14} \mathrm{~cm}^{-2}$, which is of the same order as the electron areal concentration expected in the Dirac cone. The denominator in Eq. (5) for $n_{H}$ can so be very small and would yield apparent areal concentrations much larger than the actual ones. The fact that Hall voltage sign inversion is not observed suggests that $2 \mathrm{D}$ electron mobility is much higher than measured Hall mobility at $5 \mathrm{GPa}$. This conclusion is consistent with the value of $1000 \mathrm{~cm}^{2} / \mathrm{Vs}$ as determined by Bansal et al. ${ }^{16}$

We should also recall that $n$-type surface doping induced by oxidation, as shown by Kong et al., ${ }^{19}$ cannot be excluded, and these extra 2D electrons could also be contributing to the high electron areal concentrations measured at high pressure. These surface subbands have parabolic $\mathrm{E}(\mathrm{k})$ dispersion, and their density of states would be constant. Considering Fermi-level energies at some $0.2 \mathrm{eV}$ above the subband minimum, ${ }^{20,21}$ the contribution of these subbands to the surface electron areal concentration would be larger than $10^{13} \mathrm{~cm}^{-2}$, that is of the same order of magnitude of the expected areal concentration in the TI system for samples from the A ingot.

Hall-effect experiments at high pressure and low temperature should provide further data to determine the transport parameters of the different types of carriers contributing to charge transport in $\mathrm{Bi}_{2} \mathrm{Se}_{3}$.

\section{CONCLUSIONS}

In summary, we have shown that the optical gap of $\mathrm{Bi}_{2} \mathrm{Se}_{3}$ increases under pressure, in agreement with the increase of the direct gap at the $\Gamma$ point, as determined by $a b$ initio band structure calculations. A large increase of the electronic dielectric constant is also observed and attributed to the decrease under pressure of the Penn gap that is tentatively assigned to higher-energy-allowed transitions in the $\Gamma-\mathrm{L}$ direction. We have also shown the occurrence of a pressuredriven 3D electron trapping mechanism that reduces the bulk electron concentration in $\mathrm{Bi}_{2} \mathrm{Se}_{3}$ and has been assigned to a shallow-to-deep transformation of donor levels, associated with a change in the ordering of the conduction band minima can provide an alternative method to isolate and determine the transport contribution of 2D electrons in the Dirac cones. In the conditions of results here reported, this contribution could not be fully isolated because of the presence of minority holes or oxidation-induced extra $n$-type surface doping. Lowtemperature measurements under high pressure should prevent the excitation of minority holes and create the ideal conditions in which $\mathrm{Bi}_{2} \mathrm{Se}_{3}$ transport properties are fully controlled by surface 2D electrons.

\section{ACKNOWLEDGMENTS}

This work has been done under financial support from Spanish MICINN under Grants No. MAT2008-06873-C0202, No. MAT2007-66129, No. MAT2010-21270-C04-03/04, No. CSD2007-00045, and Prometeo No. GV2011/035. The supercomputer time has been provided by the Red Española de Supercomputación (RES) and the MALTA cluster.
*Corresponding author: alfredo.segura@uv.es

${ }^{1}$ S. K. Mishra, S. Satpathy, and O. Jepsen, J. Phys.: Condens. Matter 9, 461 (1997).

${ }^{2}$ Y. S. Hor, A. Richardella, P. Roushan, Y. Xia, J. G. Checkelsky, A. Yazdani, M. Z. Hasan, N. P. Ong, and R. J. Cava, Phys. Rev. B 79, 195208 (2009).

${ }^{3}$ H. Zhang, C. X. Liu, X. L. Qi, X. Dai, Z. Fang, and S. C. Zhang, Nat. Phys. 5, 438 (2009).

${ }^{4}$ M. Z. Hassan and C. L. Kane, Rev. Mod. Phys. 82, 3045 (2010).

${ }^{5}$ J. E. Moore, Nature (London) 464, 194 (2010).

${ }^{6}$ Y. Xia, D. Qian, D. Hsieh, L. Wray, A. Pal, H. Lin, A. Bansil, D. Grauer, Y. S. Hor, R. J. Cava, and M. Z. Hasan, Nat. Phys. 5, 398 (2009).

${ }^{7}$ Y. L. Chen, J. G. Analytus, J.-H. Chu, Z. K. Liu, S.-K. Mo, X. L. Qi, H. J. Zhang, D. H. Lu, X. Dai, Z. Fang, S.-C. Zhang, I. R. Fisher, Z. Hussain, and Z.-X. Shen, Science 325, 178 (2009).

${ }^{8}$ D. Hsieh, Y. Xia, D. Qian, L. Wray, J. H. Dil, F. Meier, J. Osterwalder, L. Patthey, J. G. Checkelsky, N. P. Ong, A. V. Fedorov, H. Lin, A. Bansil, D. Grauer, Y. S. Hor, R. J. Cava, and M. Z. Hasan, Nature (London) 460, 1101 (2009).
${ }^{9}$ Z. Alpichshev, J. G. Analytis, J. H. Chu, I. R. Fisher, Y. L. Chen, Z. X. Shen, A. Fang, and A. Kapitulnik, Phys. Rev. Lett. 104, 016401 (2010).

${ }^{10}$ P. Roushan, J. Seo, C. V. Parker, Y. S. Hor, D. Hsieh, D. Qian, A. Richardella, M. Z. Hasan, R. J. Cava, and A. Yazdani, Nature (London) 460, 1106 (2009).

${ }^{11}$ N. P. Butch, K. Kirshenbaum, P. Syers, A. B. Sushkov, G. S. Jenkins, H. D. Drew, and J. Paglione, Phys. Rev. B 81, 241301(R) (2010).

${ }^{12}$ Z. Wang, T. Lin, P. Wei, X. Liu, R. Dumas, K. Liu, and J. Shi, Appl. Phys. Lett. 97, 042112 (2010).

${ }^{13}$ Zhi Ren, A. A. Taskin, Satoshi Sasaki, Kouji Segawa, and Yoichi Ando, Phys. Rev. B 82, 241306(R) (2010).

${ }^{14}$ V. A. Kulbachinskii, N. Miura, H. Nakagawa, H. Arimoto, T. Ikaida, P. Lostak, and C. Drasar, Phys. Rev. B 59, 15733 (1999).

${ }^{15}$ J. G. Analytis, R. D. McDonald, S. C. Riggs, Jiun-Haw Chu, G. S. Boebinger, and I. R. Fisher, Nat. Phys. 6, 960 (2010).

${ }^{16}$ N. Bansal, Y. S. Kim, M. Brahlek, E. Edrey, and S. Oh, e-print arXiv:1104.5709.

${ }^{17}$ S. Cho, N. P. Butch, J. Paglione, and Mi. S. Fuhrer, Nano Lett. 11, 1925 (2011). 
${ }^{18}$ Y. Zhang, K. He, C. Z. Chang, C. L. Song, L. L. Wang, X. Chen, J. F. Jia, Z. Fang, X. Dai, W. Y. Shan, S. Q. Shen, Q. Niu, X. L. Qi, S. C. Zhang, X. C. Ma, and Q. K. Xue, Nat. Phys. 6, 584 (2010).

${ }^{19}$ D. Kong, J. J. Cha, K. Lai, H. Peng, J. G. Analytis, S. Meister, Y. Chen, H.-J. Zhang, I. R. Fisher, Z.-X. Shen, and Y. Cui, ACS Nano 5, 4698 (2011).

${ }^{20}$ H. M. Benia, C. Lin, K. Kern, and C. R. Ast, Phys. Rev. Lett. 107, 177602 (2011).

${ }^{21}$ P. D. C. King, R. C. Hatch, M. Bianchi, R. Ovsyannikov, C. Lupulescu, G. Landolt, B. Slomski, J. H. Dil, D. Guan, J. L. Mi, E. D. L. Rienks, J. Fink, A. Lindblad, S. Svensson, S. Bao, G. Balakrishnan, B. B. Iversen, J. Osterwalder, W. Eberhardt, F. Baumberger, and Ph. Hofmann, Phys. Rev. Lett. 107, 096802 (2011).

${ }^{22}$ J. J. Hamlin, J. R. Jeffries, N. P. Butch, P. Syers, D. A. Zocco, S. T. Weir, Y. K. Vohra, J. Paglione, and M. B. Maple, J. Phys.: Condens. Matter 24, 035602 (2012).

${ }^{23}$ H. Köhler and J. Hartmann, Phys. Status Solidi B 63, 171 (1974).

${ }^{24}$ V. Panchal, A. Segura, and J. Pellicer-Porres, High Press. Res. 31, 445 (2011).

${ }^{25}$ J. C. Chervin, B. Canny, J. M. Besson, and P. Pruzan, Rev. Sci. Instrum. 66, 2595 (1995).

${ }^{26}$ G. J. Piermarini, S. Block, J. D. Barnett, and R. A. Forman, J. Appl. Phys. 46, 2774 (1975).

${ }^{27}$ D. Errandonea, A. Segura, D. Martínez-García, and V. Muñoz-San Jose, Phys. Rev. B 79, 125203 (2009).

${ }^{28}$ D. Errandonea, D. Martinez-Garcia, A. Segura, J. Ruiz-Fuertes, R. Lacomba-Perales, V. Fages, A. Chevy, L. Roa, and V. Muñoz-San Jose, High Press. Res. 26, 513 (2006).

${ }^{29}$ P. Hohenberg and W. Kohn, Phys. Rev. 136, B864 (1964).

${ }^{30}$ G. Kresse and J. Hafner, Phys. Rev. B 47, 558 (1993); 49, 14251 (1994); G. Kresse and J. Furthmüller, Comput. Mater. Sci. 6, 15 (1996); Phys. Rev. B 54, 11169 (1996).
${ }^{31}$ P. E. Blöchl, Phys. Rev. B 50, 17953 (1994); G. Kresse and D. Joubert, ibid. 59, 1758 (1999).

${ }^{32}$ J. P. Perdew, A. Ruzsinszky, G. I. Csonka, O. A. Vydrov, G. E. Scuseria, L. A. Constantin, X. Zhou, and K. Burke, Phys. Rev. Lett. 100, 136406 (2008).

${ }^{33}$ A. Mujica, A. Rubio, A. Muñoz, and R. J. Needs, Rev. Mod. Phys. 79, 863 (2003)

${ }^{34}$ H. Köhler and C. Becker, Phys. Status Solidi B 61, 533 (1974),

${ }^{35}$ R. Vilaplana, D. Santamaría-Pérez, O. Gomis, F. J. Manjón, J. González, A. Segura, A. Muñoz, P. Rodríguez-Hernández, E. Pérez-González, V. Marín-Borrás, V. Muñoz-Sanjose, C. Drasar, and V. Kucek, Phys. Rev. B 84, 184110 (2011).

${ }^{36}$ A. D. LaForge, A. Frenzel, B. C. Pursley, Tao Lin, Xinfei Liu, J. Shi, and D. N. Basov, Phys. Rev. B 81, 125120 (2010).

${ }^{37}$ Y. Sharma and P. Srivastava, 5th International Conference on Themophysical Properties, AIP Conference Proceedings Vol. 1249 (American Institute of Physics, Melville, New York, 2010), pp. 183-187.

${ }^{38}$ D. R. Penn, Phys. Rev. 128, 2093 (1962).

${ }^{39}$ J. C. Phillips, Rev. Mod. Phys. 42, 317 (1970).

${ }^{40}$ J. A. Van Vechten, Phys. Rev. 182, 891 (1969).

${ }^{41}$ J. A. Van Vechten, Phys. Rev. 187, 1007 (1969).

${ }^{42}$ P. Larson, V. A. Greanya, W. C. Tonjes, Rong Liu, S. D. Mahanti, and C. G. Olson, Phys. Rev. B 65, 085108 (2002).

${ }^{43}$ J. Chang, P. Jadaun, L. F. Register, S. K. Banerjee, and B. Sahu, Phys. Rev. B 84, 155105 (2011).

${ }^{44}$ T. Suski, R. Piotrzkowski, P. Wiśniewski, E. Litwin-Staszewska, and L. Dmowski, Phys. Rev. B 40, 4012 (1989).

${ }^{45}$ D. Errandonea, A. Segura, J. F. Sánchez-Royo, V. Muñoz, P. Grima, A. Chevy, and C. Ulrich, Phys. Rev. B 55, 16217 (1997).

${ }^{46}$ J. G. Analytis, J.-H. Chu, Y. Chen, F. Corredor, R. D. McDonald, Z. X. Shen, and I. R. Fisher, Phys. Rev. B 81, 205407 (2010).

${ }^{47}$ H. Köhler and H. Fabricius, Phys. Status Solidi B 71, 487 (1975). 